\title{
Disease activity is associated with LV dysfunction in rheumatoid arthritis patients without clinical cardiovascular disease
}

\author{
Punchong Hanvivadhanakul ${ }^{1 *+}$ and Adisai Buakhamsri ${ }^{2+}$
}

\begin{abstract}
Objectives: The cross-sectional study aimed to assess left ventricular systolic function using global longitudinal strain (GLS) by speckle-tracking echocardiography (STE) and arterial stiffness using cardio-ankle vascular index (CAVI) in Thai adults with rheumatoid arthritis (RA) and no clinical evidence of cardiovascular disease (CVD).

Methods: Confirmed RA patients were selected from a list of outpatient attendees if they were 18 years $(\mathrm{y})$ without clinical, ECG and echocardiographic evidence of CVD, diabetes mellitus, chronic kidney disease, and excess alcoholic intake. Controls were matched with age and sex to a list of healthy individuals with normal echocardiograms. All underwent STE and CAVI.

Results: 60 RA patients (females $=55$ ) were analysed. Mean standard deviation of patient and control ages were $50 \pm$ 10.2 and $51 \pm 9.9 \mathrm{y}$, respectively, and mean duration of RA was $9.0 \pm 6.8 \mathrm{y}$. Mean DAS28-CRP and DAS28-ESR were $2.9 \pm$ 0.9 and $3.4 \pm 0.9$, respectively. There was no between-group differences in left ventricular ejection fraction (LVEF), LV sizes, LVMI, LV diastolic function and CAVI were within normal limits but all GLSs values was significantly lower in patients vs. controls: $17.6 \pm 3.4$ vs $20.4 \pm 2.2(p=0.03)$. Multivariate regression analysis demonstrated significant correlations between GLSs and RA duration ( $p=0.02)$, and GLSs and DAS28-CRP $(p=0.041)$.

Conclusions: Patients with RA and no clinical CV disease have reduced LV systolic function as shown by lower GLSs. It is common and associated with disease activity and RA disease duration. 2D speckle-tracking GLSs is robust in detecting this subclinical LV systolic dysfunction.
\end{abstract}

Keywords: Rheumatoid arthritis, Subclinical LV dysfunction, Global longitudinal strain, DAS28-CRP, Disease duration

\section{Introduction}

Rheumatoid arthritis (RA) is a systemic autoimmune disease, characterised by chronic joint inflammation and destruction. Extra-articular organ involvement, such as the skin, eyes, heart, lungs, and blood vessels may also cause clinically significant pathology and affect clinical outcomes and quality of life. Compared to the general population, patients with RA have a $8-15$ year reduced life expectancy [1] due principally to cardiovascular complications, most commonly atherosclerotic diseases

\footnotetext{
* Correspondence: phanvivad@gmail.com

†Punchong Hanvivadhanakul and Adisai Buakhamsri contributed equally to the study.

${ }^{1}$ Division of Rheumatology, Department of Medicine, Faculty of Medicine, Thammasat University, 99/209 Moo 18, Paholyothin Road, Klong Luang, Pathumthanee 12120, Thailand

Full list of author information is available at the end of the article
}

and congestive heart failure (CHF) [2]. Although the pathophysiologic mechanism is not clearly defined, the chronic inflammatory state of RA itself seems to play a pivotal role in both vascular changes and impaired myocardial function $[3,4]$. Previous studies have shown that asymptomatic left ventricular myocardial dysfunction is common, both systolic and diastolic abnormalities at rates of; 45 and 31\% respectively [5-10]. Some studies have also shown increased left ventricular mass in RA patients $[5,11,12]$. However, most of the studies were conducted in western and middle eastern countries. There are few studies from Asian populations and the outcomes differ $[13,14]$. Thus, the present study was conducted to determine left ventricular structure and function and arterial wall stiffness. Specifically, we used left ventricular deformation (global longitudinal strain or

(C) The Author(s). 2019 Open Access This article is distributed under the terms of the Creative Commons Attribution 4.0 International License (http://creativecommons.org/licenses/by/4.0/), which permits unrestricted use, distribution, and reproduction in any medium, provided you give appropriate credit to the original author(s) and the source, provide a link to the Creative Commons license, and indicate if changes were made. The Creative Commons Public Domain Dedication waiver (http://creativecommons.org/publicdomain/zero/1.0/) applies to the data made available in this article, unless otherwise stated. 
GLS) to assess subclinical LV systolic dysfunction [15] and measured the cardio-ankle vascular index (CAVI) to evaluate arterial stiffness (LV afterload). CAVI is a simple bedside tool to estimate the combined stiffness of the aorta, iliac, femoral, and tibial arteries. Arterial stiffness may affect the structure and function of the left ventricle [16]. We hypothesized that GLSs and CAVI may be associated with LV functional and structural change, respectively, in patient with RA and no clinical CV disease.

\section{Patients and methods}

\section{Study population}

This was a cross-sectional study of patients with proven rheumatoid arthritis who had been followed up at the Rheumatology clinic of Thammasat University Hospital (TUH) between August 2015 and July 2017. All underwent a medical history, thorough physical examination, and medical record review to assess their eligibility for the study. To be included, they had to: (i) be at least 18 years (y) old, (ii) have fulfilled the 1987 American College of Rheumatology (ACR) classification criteria [17] or the $2010 \mathrm{ACR} /$ European League Against Rheumatism (EULAR) classification RA criteria [18], (iii) have been under outpatient follow-up for $\geq 2 y$, and (iv) have given written informed consent. Patients were excluded if they had one of the following: arterial hypertension, coronary heart disease, valvular heart disease, CHF, diabetes mellitus, chronic kidney disease, aortic/peripheral arterial disease, clinically significant arrhythmia or alcoholic intake.

\section{Control subjects}

Control subjects were obtained from our echocardiography database of 100 healthy normal subjects, previously enrolled in an unrelated echocardiography study. They were declared healthy if they had normal findings in all following aspects: medical history, physical examination and no current intake of any medication. Additionally, 12-lead electrocardiogram and comprehensive echocardiogram must be normal. They were then ageand sex-matched with the RA patients. Age-matching was limited at $+/-2$ years. This study was approved by the Ethics Committee of the Faculty of Medicine, Thammasat University. Informed consent was obtained from all participants included in the study.

\section{Clinical data collection}

Patient data were recorded on to standard case report forms and included age, sex, body mass index, smoking status, waist circumference, and pertinent physical signs (e.g. locations of tender and/or swollen joints). Disease activity and functional status were assessed by the Disease Activity Score 28 (DAS28), C-reactive protein (CRP), erythrocyte sedimentation rate (ESR) and a health assessment questionnaire (HAQ). The medical records were also reviewed to determine disease duration, medication use, cumulative dosage of corticosteroids and methotrexate, and radiographic joint erosions.

\section{Patient blood sampling}

After $12 \mathrm{~h}$ of overnight fasting, blood was drawn to determine the following laboratory investigations: fasting blood glucose, lipid profile (total cholesterol, triglyceride, high-density lipoprotein, and low-density lipoprotein), serum creatinine, calculated glomerular filtration rate (GFR), CRP, ESR, rheumatoid factor (RF), and anticitrullinated peptide antibody (ACPA). RF was detected by latex agglutination (Plasmatec Laboratory Co, Cambridge, UK) and serum ACPA by immunofluorescence (Thermo Fisher Scientific EliA, Germany); an ACPA level $\geq 5.6 \mathrm{IU} / \mathrm{mL}$ was positive, according to the manufacturer's instructions.

\section{Echocardiograms}

Transthoracic two-dimensional (2-D) echocardiography was performed in all participants with an iE33 ultrasound machine equipped with an S5 transducer (Philips medical imaging, Andover, MA). Standard echocardiographic apical and short-axis views were obtained in accordance with the American Society of Echocardiography and European Association of Cardiovascular Imaging recommendations [19]. 2-D images were carefully adjusted to avoid over gain, which may affect the distance measurement used for LV mass calculation. Second-harmonic images were acquired for speckle tracking imaging. With high frame rate B-mode scans (40-90 frames/s) Pulsed-wave Doppler imaging were also obtained in the apical four-chamber view for assessment of the septal and lateral mitral annulus (Ea). These images with at least 3 loops of each apical LV view were stored in a digital cine-loop format for off-line analysis.

Left ventricular mass (LVM) was calculated by two different methods, as recommended by the American and European guidelines [19]: (i) M-mode tracing of the LV in the parasternal long-axis and cube formula, and (ii) 2$\mathrm{D}$ method using the LV short-axis view at papillary muscle level, apical four-chamber view and area-length formula. LVM values obtained from these two methods were then indexed with body surface area and reported as LV mass index (LVMI). LV hypertrophy was present if LVMI by either method exceeding a cut-off values according to gender: (i) linear method: $95-115 \mathrm{~g} / \mathrm{m}^{2}$ and (ii) 2-D method: $88-102 \mathrm{~g} / \mathrm{m}^{2}$ for women and men, respectively. All other echocardiographic data were used to determine cardiac dimensions, left ventricular systolic and diastolic functions in compliance with standard criteria $[19,20]$. 
LV longitudinal strain [LS] measurement with speckletracking technique was performed in Excelera workstation (QLAB11, Philips) by an experienced cardiologist blinded to subject group. Speckle-tracking echocardiography (STE) utilizes a software specialized for tracking acoustic markers in standard 2-D grey scale images over one cardiac cycle $[21,22]$. Strain is a parameter of myocardial deformation expressed as a negative percentage value since the left ventricle shortens along its longitudinal axis during systole. The reduced absolute strain values (less negative strain) reflect diminished contractile function. In brief, LV myocardium (region of interest) was manually traced from medial to lateral mitral annulus in each standard apical view with good 2-D image quality. The software then performed automatic tracking of echocardiographic speckles over a cardiac cycle. The tracking quality was determined visually and the process repeated if the result was poor. If the tracking was still at poor quality, we excluded that segment from calculation of mean GLS. Any patient with more than 1 segment of poor tracking quality was not enrolled to final analysis. Longitudinal-strain (LS) curve over one cardiac cycle was generated-with-measured maximal LS during systole in each view. Global longitudinal strain during systole (GLSs) was the automated average value from 2 different cardiac cycles. In this study, GLS values were reported as absolute number (higher number means more shortening of myocardium).

\section{Cardio-ankle vascular stiffness index (CAVI)}

CAVI, an index of arterial stiffness, was determined using a dedicated device (VaSera VS-2000, Fukuda Denshi, Japan). Participants laid in the supine position for at least $20 \mathrm{~min}$ before testing. Blood pressure cuffs were then applied to the upper (brachial artery) and lower (just above ankle) extremities on both sides to obtain arterial pressures by oscillometry while echocardiograms and cardiac phonograms were being recorded. Pulse wave velocity (PWV) was measured; PWV = the length from aortic valve to the ankle divided by the combination of two time intervals (time from aortic valve closing to the notch of the brachial pulse wave and time from from the rise of the brachial pulse wave to the ankle pulse wave). CAVI was automatically calculated using following equation [16]:

$$
\mathrm{CAVI}=\mathrm{a} \times\left[(2 \rho / \Delta \mathrm{P}) \times \ln (\mathrm{Ps} / \mathrm{Pd}) \times \mathrm{PWV}^{2}\right]+\mathrm{b}
$$

where $\rho$ is blood density, $\Delta \mathrm{P}$ is 'Ps-Pd', $\mathrm{Ps} / \mathrm{Pd}$ are systolic/diastolic pressures and $\mathrm{a} / \mathrm{b}$ are constants.

According to the manufacturer's instructions, there are 3 categories of CAVI value; normal $(\mathrm{CAVI}<8.0)$, borderline (CAVI $=8.0-<9.0)$ and abnormal (CAVI $\geq 9.0)$. All measurements were performed.

\section{Reproducibility of LVM and GLSs measurements}

Echocardiographic data sets of 12 patients (corresponding to $10 \%$ of the studied population) were randomly chosen to assess intra- and interobserver variability of LVMI and GLSs. The measurements were performed twice by the same observer separated by 1-week interval and by a second observer blinded to the results of the first observer. Measurement variabilities were assessed using intraclass correlation coefficients. The intraobserver correlation coefficients for LVMI and GLSs were 0.92 and 0.95 respectively and those of LVMI and GLSs were 0.88 and 0.92 , respectively. Thus, overall reproducibility was good.

\section{Statistical analysis}

Continuous variables were expressed as mean \pm standard deviation (SD) and categorical variables as frequencies. Between-group comparisons were performed using Wilcoxon rank sum tests, Chi-square test or Fisher's exact test where appropriate. Multiple regression analysis, adjusted for age, gender, blood pressure, BMI, heart rate and LVMI (where appropriate) were used to examine associations between LVMI or global LV strain (in separate model) and characteristics of RA disease. The correlation of CAVI and GLSs was assessed with Pearson's correlation coefficient. All tests were two-sided and a value of $\leq 0.05$ was considered statistically significant. Data analysis was performed using SPSS software version 14.0 (SPSS Inc., Chicago, Illinois).

\section{Results}

Two hundred and eighty patients with RA have been screened and 62 were initially enrolled but two were later excluded from the final analysis because: (i) one male patient had hypertension diagnosed during the study even though prior BP recordings at study enrolment were normal. Hypertension was confirmed by 24-h ambulatory blood pressure monitoring. He had echocardiographic-LVH (LVMI $122 \mathrm{~g} / \mathrm{m}^{2}$ and $110 \mathrm{~g} / \mathrm{m}^{2}$ by M-mode and 2-D, respectively) and borderline CAVI (8.8), and (ii) a female who was lost to follow-up; her echocardiogram and CAVI were within normal limits. General clinical characteristics are presented in Table 1. Features of RA patients are detailed in Table 2. The important echocardiographic findings including LVMI, GLSs and CAVI (in the patient group) are shown in Tables 3 and 4.

\section{Patients with rheumatoid arthritis}

The 60 RA patients (female $=55$ ) had a mean age of $51 \pm 9.9$ y; most were non-smokers (95\%). Dyslipidemia and impaired fasting glucose were present in $18(30 \%)$ and $8(13 \%)$ patients, respectively, but all had normal eGFR values. Mean (range) RA duration was 7 (2-32) y. 
Table 1 Clinical characteristics of patients with rheumatoid arthritis and age/sex-matched healthy controls

\begin{tabular}{llll}
\hline Clinical characteristics & Patients $n=60$ & Controls $n=60$ & $P$-value \\
\hline Age (years) & $50 \pm 10.2$ & $51 \pm 9.9$ & 0.8 \\
Women & $55(92 \%)$ & $55(92 \%)$ & 1.0 \\
BMI $\left(\mathrm{kg} / \mathrm{m}^{2}\right)$ & $22 \pm 3$ & $21 \pm 2.5$ & 0.64 \\
Systolic blood pressure $(\mathrm{mmHg})$ & $120 \pm 9.7$ & $117 \pm 8.8$ & 0.5 \\
Diastolic blood pressure $(\mathrm{mmHg})$ & $75 \pm 7.7$ & $73 \pm 10$ & 0.2 \\
Heart rate (BPM) & $74 \pm 7$ & $77 \pm 9$ & 0.6 \\
Smoking history & $3(5 \%)$ & None & $<0.01$ \\
Dyslipidemia & $17(28 \%)$ & None & $<0.01$ \\
\hline
\end{tabular}

No patients had rheumatoid nodule. Biological agent was not used in our patient cohort. Rheumatoid factor (RF) was positive in 32 (54\%) patients and anticitrullinated peptide antibody (ACPA) in 43 (72\%) patients; both were positive in $31(52 \%)$ patients. The DAS28-CRP, DAS28-ESR and HAQ scores were $2.9 \pm 0.9$, $3.4 \pm 1.0$ and $0.5 \pm 0.6$, respectively.

\section{Cardiac geometry and function}

Cardiac chamber sizes including the left ventricle and left atrium were normal in all patients. LVEF and dopplerbased diastolic function were also normal by the American Society of Echocariography criteria [23, 24]. Estimated left atrial pressure was normal and there was no echocardiographic evidence of pulmonary hypertension in all patients.

Table 2 Clinical characteristics of 60 patients with rheumatoid arthritis and regression analysis for factors associated with systolic global longitudinal strain

\begin{tabular}{|c|c|c|c|}
\hline Clinical parameters & Value & Adjusted univariate coefficient ${ }^{a}$ (SE) & $P$-value \\
\hline \multicolumn{4}{|l|}{ Clinical } \\
\hline Duration of RA (years) & $9.0 \pm 6.8(2-40)$ & $1.68(0.76)$ & 0.02 \\
\hline HAQ disability index & $0.55 \pm 0.6(0-2.25)$ & $1.1(0.49)$ & 0.58 \\
\hline Rheumatoid nodule & 0 & NA & NA \\
\hline DAS28-CRP & $2.9 \pm 0.9(1.48-5.66)$ & $1.44(0.72)$ & 0.041 \\
\hline DAS28-ESR & $3.4 \pm 0.9(1.05-6.25)$ & $0.67(0.43)$ & 0.7 \\
\hline Joint erosion & $25(42 \%)$ & $0.8(0.62)$ & 0.09 \\
\hline \multicolumn{4}{|l|}{ Medications } \\
\hline Methotrexate & $58(96 \%)$ & $0.03(0.01)$ & 0.79 \\
\hline Cumulative dose of methotrexate (g) & $2.9 \pm 1.4(0-5.36)$ & $0.26(0.02)$ & 0.47 \\
\hline Sulfasalazine & $30(50 \%)$ & $0.72(0.13)$ & 0.61 \\
\hline Hydroxychloroquine & $18(23 \%)$ & $0.4(0.27)$ & 0.53 \\
\hline Leflunomide & $20(30 \%)$ & $0.74(0.81)$ & 0.8 \\
\hline Other DMARDs & $31(52 \%)$ & $0.1(0.05)$ & 0.72 \\
\hline Corticosteriod & $29(48 \%)$ & $0.7(0.49)$ & 0.93 \\
\hline Cumulative dose of corticosteroid (g) & $2.8 \pm 4.6(0-23)$ & $0.33(0.09)$ & 0.82 \\
\hline NSAIDs & $13(22 \%)$ & $0.92(0.55)$ & 0.44 \\
\hline \multicolumn{4}{|l|}{ Laboratory } \\
\hline $\mathrm{CRP}^{\mathrm{b}}(\mathrm{mg} / \mathrm{dL})$ & $10.1 \pm 15(0.3-65.2)$ & $0.52(0.38)$ & 0.56 \\
\hline $\mathrm{ESR}^{\mathrm{b}}(\mathrm{mm} / \mathrm{hr})$ & $30 \pm 19(3-81)$ & $0.81(0.62)$ & 0.7 \\
\hline Positive RF or ACPA & 45 (75\%) & $0.59(1.0)$ & 1.0 \\
\hline
\end{tabular}

Continuous data are expressed as mean \pm SD (min-max), categorical data as $\mathrm{n}(\%)$

ACPA Anti-citrullinated peptide antibodies, CRP C-reactive protein, DAS28 Disease Activity Score 28, DMARDs Disease-modifying antirheumatic drugs, ESR Erythrocyte sedimentation rate, $H A Q$ Health assessment questionnaire, RA Rheumatoid arthritis, RF Rheumatoid factor, NA Not available or not applicable ${ }^{a}$ Adjusted for age, gender, blood pressure, body mass index, heart rate and left ventricular mass index

bog-transformed 
Table 3 Disease activity scores in patients with rheumatoid arthritis

\begin{tabular}{lll}
\hline Disease activity & $\begin{array}{l}\text { DAS28-CRP } \\
\text { N (\%) }\end{array}$ & $\begin{array}{l}\text { DAS28-ESR } \\
\text { N }(\%)\end{array}$ \\
\hline Remission $(<2.6)$ & $26(43)$ & $12(20)$ \\
Low $(2.6$ to $<3.2)$ & $10(16)$ & $13(22)$ \\
Moderate $(3.2$ to $<5.1)$ & $23(38)$ & $33(55)$ \\
Severe $(\geq 5.1)$ & $1(3)$ & $2(3)$ \\
\hline
\end{tabular}

\section{LV mass index (LVMI)}

LVMI was slightly higher with M-mode than the 2-D method. Men had higher LVMI than women subjects, However, all patients had normal LVMI independent of measurement methods. No correlation was found between LVMI and other variables of RA disease, blood pressure and CAVI.

\section{Systolic global longitudinal strain (GLSs)}

GLSs were normal for all patients when using the general cut-off point of normal limit $[23,25,26]$ but were significantly lower than the controls: $17.6 \pm 3.4 \%$ vs. $20.4 \pm 2.2 \%(p=0.03)$. Duration of rheumatoid disease and DAS28-CRP score were significantly associated with lower absolute value of GLSs after adjustment for age, gender, blood pressure, body mass index, heart rate and left ventricular mass index (univariate coefficient (SE); 1.68 (0.76), $p=0.02$ for duration of disease and 1.44 (0.72), $p=0.041$ for DAS28-CRP).

\section{Cardio-ankle velocity index (CAVI)}

CAVIs (Table 5) were normal in almost all patients $(96 \%)$ while a few were in borderline category (4\%) and
Table 5 Cardio-Ankle vascular stiffness index in patients with rheumatoid arthritis

\begin{tabular}{lll}
\hline CAVI category & Value $^{a}$ & N (\% of total) \\
\hline Normal $(<8)$ & $6.7 \pm 1.5$ & $58(96)$ \\
Borderline $(8$ to $<9)$ & $8.5 \pm 4.2$ & $2(4)$ \\
Abnormal $(\geq 9)$ & $\mathrm{NA}$ & $0(0)$
\end{tabular}

${ }^{\mathrm{a}}$ Mean $\pm \mathrm{SD}$, categorical data as $\mathrm{n}(\%)$

none had abnormal CAVI. There was no correlation between CAVI and GLSs $(r=-0.13, p=0.6)$.

\section{Discussions}

We have demonstrated that LV systolic function of patient with RA is worse than those of matched healthy subject. This subclinical LV systolic dysfunction as shown by lower GLSs (although still in normal range) is common in patients with chronic RA and independently associated with disease activity and RA duration. All RA patients in our study underwent prospectively comprehensive echocardiographic examinations. Cardiac geometry (i.e. chamber sizes) and all echocardiographic parameters of systolic/diastolic function (except GLSs) were within normal limits and were not significantly different from those of the control subjects. We did not find $\mathrm{LVH}$ (determined by LVMI) in our RA patients even when using two different methods of assessment. This contrasts with other studies which have reported high rates of increased LVM/LVMI in RA patients [12, 27,28 . In general, significant proportions of RA patients have co-existing cardiovascular risk factors such as hypertension or an increased Framingham Risk Score, which have been reported in up to $40 \%$ of RA patients [13].

Table 4 Echocardiographic parameters and systolic global longitudinal strain of patients with rheumatoid arthritis and age and sex-matched control subjects

\begin{tabular}{|c|c|c|c|}
\hline Parameters & Patients & Controls & $P$-value \\
\hline LV end-diastolic volume index ${ }^{a}\left(\mathrm{~mL} / \mathrm{m}^{2}\right)$ & $52 \pm 8$ & $54 \pm 7$ & 0.10 \\
\hline LV end-systolic volume index ${ }^{a}\left(\mathrm{~mL} / \mathrm{m}^{2}\right)$ & $22 \pm 5$ & $24 \pm 4$ & 0.38 \\
\hline LV ejection fraction ${ }^{a}$ & $57 \pm 4$ & $56 \pm 3$ & 0.50 \\
\hline Left atrial volume index $\left(\mathrm{mL} / \mathrm{m}^{2}\right)$ & $26 \pm 6$ & $24 \pm 5$ & 0.67 \\
\hline LV mass index (gm/m²) (M-mode) & $74 \pm 8$ & $76 \pm 12$ & 0.40 \\
\hline LV mass index (gm/m²) (2D-method) & $72 \pm 7$ & $75 \pm 10$ & 0.09 \\
\hline LV hypertrophy & 0 & 0 & NS \\
\hline LV diastolic dysfunction ${ }^{c}$ & 0 & 0 & NS \\
\hline Average $E^{\prime}$ (septal and lateral) (cm/s) & $12.4 \pm 2.5$ & $13.1 \pm 3.2$ & 0.2 \\
\hline$E / E^{\prime}$ & $6.2 \pm 1.6$ & $6.8 \pm 2.1$ & 0.35 \\
\hline Systolic global longitudinal strain (\%) & $17.6 \pm 3.4$ & $20.4 \pm 2.2$ & 0.03 \\
\hline
\end{tabular}

$E^{\prime}$ Mitral annulus tissue velocity curing early diastole, $E$ Peak mitral inflow velocity during early diastole NS Not significant

${ }^{a}$ Modified Simpson's method

${ }^{b}$ According to 2015 American Society of Echocardiography: recommendations for cardiac chamber quantification by echocardiography

'According to 2016 American Society of Echocardiography: recommendations for the evaluation of left ventricular diastolic function by echocardiography 
This study was meticulously designed to avoid confounding factors that affect LV mass, like arterial hypertension, diseases associated with LV pressure/volume loading and hypertrophic/infiltrative cardiomyopathy. These had been ruled out by reviewing medical records, conducting detailed physical examinations, ECGs and echocardiograms prior to enrolment. Importantly, we also performed CAVI in all patients to detect abnormal arterial stiffness which can be present in chronic inflammatory diseases like RA [29-31]. CAVI has been used to assess arterial stiffness in subjects at risk of or with clinically overt cardiovascular disease [32, 33]; increased arterial stiffness is related to changes in LV geometry and LV mass independent of arterial blood pressure [34-36]. However, CAVI results in our study were mostly normal with no patient having an abnormal CAVI. This together with strict study inclusion criteria, may partly explain the absence of $\mathrm{LVH}$ in our patients i.e. $\mathrm{LVH}$ as defined by LVMI higher than normal limits, is uncommon in RA patients if conditions causing increased LV afterload are absent.

Conventional parameters of LV systolic and diastolic function in our patients were also normal. We also used GLSs to assess subclinical changes of LV systolic function and found that even though our RA patients had significantly lower values vs. controls, all values were within normal limits (higher than 15.9\%) [25] and were independent of LV diastolic function. Since GLSs is more sensitive to change in LV systolic function than LVEF, our findings demonstrate subtle LVSD in RA. GLSs, as a marker of LVSD, has previously been shown to be abnormal in early stage heart disease and have prognostic significance in many cardiac disorders such as chemotherapy-related cardiomyopathy [37] and hypertrophic cardiomyopathy [38].

Our results were also in line with a study in patients with RA by Fine et al. [9] who demonstrated a reduction of LV longitudinal strain independent of LVEF and the degree of LV diastolic dysfunction. We identified DAS28-CRP and duration of disease as independent factors affecting subclinical LVSD; both factors are indicative of the severity and duration of inflammation. Using a simplified Disease Activity Index of $<3.3$, Midtbø et al. [39] have shown that when RA is in remission, patients have improved LV function (assessed by stress corrected mid-wall shortening and global longitudinal strain) compared to patients with active disease. Proposed mechanisms of LV systolic dysfunction in RA include chronic inflammation, oxidative stress and myocyte dysfunction, interstitial fibrosis and endothelial dysfunction that leads to impaired perfusion [3]. These can reasonably explain our findings that disease activity determined subclinical LV systolic dysfunction in our patient cohort.
Analysis model of current study showed that factors such as age, use of DMARDs or corticosteroids and inflammatory markers were not associated with LVSD. Their effects may have been masked as they were likely incorporated into DAS28-CRP and duration of disease. In addition, the ESR, which may be influenced by many factors and is a less sensitive marker of RA disease activity compared to CRP $[40,41]$. Methotrexate and prednisolone were commonly prescribed; thus, the association of these drugs to LVSD may not be able to demonstrate.

As our studied population were Asian, whether ethnic difference plays any role on the final results are yet to be determined. General consideration deserves to be mentioned. First, prevalence of $\mathrm{CV}$ risk factors in Asians with RA appears to be lower than that of non-Asian population (US and European population) [42]. These risk factors including hypertension, diabetes, dyslipidemia and central obesity (or, collectively, metabolic syndrome) have direct and indirect insult to myocardial fu nction. Second, geometric changes of the heart due to those underlying disease and/or races may have impact on left ventricular function [43]. Third, genetic susceptibility may increases disease severity and chronicity of systemic inflammation. Although it was not well established in RA, genetic susceptibilities specific to some ethnic groups were demonstrated in other rheumatic diseases such as SLE [44, 45]. Fourth, disparity of ethnicrelated socioeconomic level (ability to access proper treatment, timely diagnosis/follow-up) may also account for the different changes of cardiovascular structure. Thus, these ethnic-related factors potentially affect LV systolic function and, inevitably, GLSs.

\section{Study limitations}

Circumferential and radial strains were not studied in our patients as these parameters are less reproducible than longitudinal strain [46]. Analysis of GLSs is dependent on quality of two-dimensional image. Poor speckle tracking will directly affect the reliability of GLS value. The power of our study to detect small change in LV geometry and function was limited by the small number of cases and controls (60/group). This, however, was the result of rigorous inclusion criteria to ascertain that the patients had no underlying CVD, which would have been a confounding factor in our study results. A small difference may not be detected if only a universal normal cut-off point was used. We did not take serial measurements, so a temporal relationship between disease activity and left ventricle function could not be demonstrated; this is an avenue of future research to see if we can replicate the results of other long term RA studies [47, 48]. Cardiac biomarkers, like cardiac troponin and NT-proBNP, were not studied in our patients 
and could have supported (or otherwise) the findings of subclinical LVSD. There were no patients with abnormal CAVIs; thus, we could not explore potential associations with cardiac function parameters. Both DAS28-ESR and DAS28-CRP may have limited power in estimation of disease activity. While DAS28 ESR reflects disease activity in the previous few weeks, DAS28-CRP does so but for a shorter term. Simplified and clinical disease activity indices (SDAI, CDAI) were not associated with LV systolic function (GLSs) in our study. Presence of smoker and dyslipidemia in patient group may possibly affect the its GLS results. We did sensitivity analysis including only those with no smoking history or dyslipidemia and found no effect on main results. However, it was unable to exclude possible effect of this two factors on GLS if studied population was larger.

\section{Conclusions}

Patients with RA and no clinical CV disease have reduced LV systolic function when comparing to that of matched healthy subjects. It is demonstrated by the lower GLSs values while conventional echocardiographic parameters of LV systolic function are still unchanged. This subclinical LV systolic dysfunction is common and associated with disease activity and RA disease duration.

\section{Clinical perspective}

2D-STE GLS can be easily performed with most echocardiogram machines. This technique provides the opportunities to identify subclinical LV systolic dysfunction in at-risk RA patients, including presence of multiple $\mathrm{CV}$ risk factors, high disease activity or long disease duration in particular. Serial GLSs measurement may be an interesting strategy and considered as a long-term follow-up tool in these patients. Further studies are needed to evaluate a proper GLSs cut-off point (or delta change); to understand mechanism(s), consequences and therapeutic implications of subclinical LV systolic dysfunction.

\footnotetext{
Abbreviations

2-D: Two-dimensional; ACPA: Anti-citrullinated peptide antibody; ACR: American College of Rheumatology; CAVI: Cardio-ankle vascular index; CHF: Congestive heart failure; CRP: C-reactive protein; CVD: Cardiovascular disease; DAS28: Disease Activity Score 28; ESR: Erythrocyte sedimentation rate; EULAR: European League Against Rheumatism; GFR: Glomerular filtration rate; GLS: Global longitudinal strain; GLSs: Global longitudinal strain during systole; HAQ: Health assessment questionnaire; LS: LV longitudinal strain; LV: Left ventricular; LVEF: Left ventricular ejection fraction; LVMI: LV mass index; LVOT: LV outflow tract; PWV: Pulse wave velocity; RA: Rheumatoid arthritis; RF: Rheumatoid factor; STE: Speckle-tracking echocardiography; TUH: Thammasat University Hospital; y: Years
}

\section{Acknowledgements}

We thank the patients and control subjects for agreeing to participate in this study. This study was supported by Thammasat university research grant (TP 2-37-2559).Dr. Bob Taylor kindly reviewed the paper.

\section{Authors' contributions}

$\mathrm{PH}$ and $\mathrm{AB}$ contribute to this study equally. PH collected, analyzed, and interpreted the patient data regarding rheumatoid arthritis. $A B$ performed echocardiogram examination, analyzed, and interpreted data. AB was a major contributor in writing the manuscript. All authors read and approved the final manuscript.

Funding

This study was supported by Thammasat university research grant (TP 2-37-2559).

\section{Availability of data and materials}

The data that support the findings of this study are available from the corresponding authors upon reasonable request.

\section{Ethics approval and consent to participate}

This study was approved by the Ethics Committee of the TUH faculty of Medicine. Informed consent was obtained from all individual participants included in the study.

\section{Consent for publication}

Not applicable.

\section{Competing interests}

The authors declare that they have no competing interests.

\section{Author details}

${ }^{1}$ Division of Rheumatology, Department of Medicine, Faculty of Medicine, Thammasat University, 99/209 Moo 18, Paholyothin Road, Klong Luang, Pathumthanee 12120, Thailand. ${ }^{2}$ Division of Cardiology, Department of Medicine, Faculty of Medicine, Thammasat University, 99/209 Moo 18, Paholyothin Road, Klong Luang, Pathumthanee 12120, Thailand.

Received: 24 May 2019 Accepted: 26 November 2019

Published online: 16 December 2019

\section{References}

1. Wolfe F, Freundlich B, Straus WL. Increase in cardiovascular and cerebrovascular disease prevalence in rheumatoid arthritis. J Rheumatol. 2003;30(1):36-40.

2. Avina-Zubieta JA, Thomas J, Sadatsafavi M, Lehman AJ, Lacaille D. Risk of incident cardiovascular events in patients with rheumatoid arthritis: a metaanalysis of observational studies. Ann Rheum Dis. 2012;71(9):1524-9.

3. England BR, Thiele GM, Anderson DR, Mikuls TR. Increased cardiovascular risk in rheumatoid arthritis: mechanisms and implications. BMJ. 2018;361: k1036.

4. Targonska-Stepniak B, Biskup M, Biskup W, Majdan M. Diastolic dysfunction in rheumatoid arthritis patients with low disease activity. Clin Rheumatol. 2019;38(4):1131-7

5. Corrao S, Salli L, Arnone S, Scaglione R, Pinto A, Licata G. Echo-Doppler left ventricular filling abnormalities in patients with rheumatoid arthritis without clinically evident cardiovascular disease. Eur J Clin Investig. 1996;26(4):293-7.

6. Wislowska M, Sypula S, Kowalik I. Echocardiographic findings, 24-hour electrocardiographic Holter monitoring in patients with rheumatoid arthritis according to Steinbrocker's criteria, functional index, value of Waaler-rose titre and duration of disease. Clin Rheumatol. 1998;17(5):369-77.

7. Liang KP, Myasoedova E, Crowson CS, Davis JM, Roger VL, Karon BL, et al. Increased prevalence of diastolic dysfunction in rheumatoid arthritis. Ann Rheum Dis. 2010;69(9):1665-70.

8. Sitia S, Tomasoni L, Cicala S, Atzeni F, Ricci C, Gaeta M, et al. Detection of preclinical impairment of myocardial function in rheumatoid arthritis patients with short disease duration by speckle tracking echocardiography. Int J Cardiol. 2012:160(1):8-14.

9. Fine NM, Crowson CS, Lin G, Oh JK, Villarraga HR, Gabriel SE. Evaluation of myocardial function in patients with rheumatoid arthritis using strain imaging by speckle-tracking echocardiography. Ann Rheum Dis. 2014;73(10):1833-9.

10. Cioffi G, Viapiana O, Ognibeni F, Dalbeni A, Gatti D, Adami S, et al, Prevalence and factors related to left ventricular systolic dysfunction in asymptomatic patients with rheumatoid arthritis. A prospective tissue Doppler echocardiography study. Herz. 2015;40(7):989-96. 
11. Wislowska M, Jaszczyk B, Kochmanski M, Sypula S, Sztechman M. Diastolic heart function in RA patients. Rheumatol Int. 2008;28(6):513-9.

12. Cioffi G, Viapiana O, Ognibeni F, Dalbeni A, Giollo A, Adami S, et al. Prevalence and factors related to inappropriately high left ventricular mass in patients with rheumatoid arthritis without overt cardiac disease. J Hypertens. 2015;33(10):2141-9.

13. Dougados M, Soubrier M, Antunez A, Balint P, Balsa A, Buch MH, et al. Prevalence of comorbidities in rheumatoid arthritis and evaluation of their monitoring: results of an international, cross-sectional study (COMORA). Ann Rheum Dis. 2014;73(1):62-8.

14. Jin S, Li M, Fang Y, Li Q, Liu J, Duan X, et al. Chinese Registry of rheumatoid arthritis (CREDIT): II. prevalence and risk factors of major comorbidities in Chinese patients with rheumatoid arthritis. Arthritis Res Ther. 2017;19(1):251.

15. Smiseth OA, Torp H, Opdahl A, Haugaa KH, Urheim S. Myocardial strain imaging: how useful is it in clinical decision making? Eur Heart J. 2016; 37(15):1196-207.

16. Sun CK. Cardio-ankle vascular index (CAVI) as an indicator of arterial stiffness. Integr Blood Press Control. 2013;6:27-38.

17. Arnett FC, Edworthy SM, Bloch DA, McShane DJ, Fries JF, Cooper NS, et al. The American Rheumatism Association 1987 revised criteria for the classification of rheumatoid arthritis. Arthritis Rheum. 1988;31(3):315-24.

18. Aletaha D, Neogi T, Silman AJ, Funovits J, Felson DT, Bingham CO 3rd, et al. 2010 rheumatoid arthritis classification criteria: an American College of Rheumatology/European League Against Rheumatism collaborative initiative. Arthritis Rheum. 2010;62(9):2569-81.

19. Lang RM, Badano LP, Mor-Avi V, Afilalo J, Armstrong A, Ernande L, et al. Recommendations for cardiac chamber quantification by echocardiography in adults: an update from the American Society of Echocardiography and the European Association of Cardiovascular Imaging. Eur Heart J Cardiovasc Imaging. 2015;16(3):233-70.

20. Nagueh SF, Smiseth OA, Appleton CP, Byrd BF 3rd, Dokainish H, Edvardsen $T$, et al. Recommendations for the evaluation of left ventricular diastolic function by echocardiography: an update from the American Society of Echocardiography and the European Association of Cardiovascular Imaging. J Am Soc Echocardiogr. 2016;29(4):277-314.

21. Reisner SA, Lysyansky P, Agmon Y, Mutlak D, Lessick J, Friedman Z. Global longitudinal strain: a novel index of left ventricular systolic function. J Am Soc Echocardiogr. 2004;17(6):630-3.

22. Langeland S, D'Hooge J, Wouters PF, Leather HA, Claus P, Bijnens B, et al. Experimental validation of a new ultrasound method for the simultaneous assessment of radial and longitudinal myocardial deformation independent of insonation angle. Circulation. 2005;112(14):2157-62.

23. Lang RM, Badano LP, Mor-Avi V, Afilalo J, Armstrong A, Ernande L, et al. Recommendations for cardiac chamber quantification by echocardiography in adults: an update from the American Society of Echocardiography and the European Association of Cardiovascular Imaging. J Am Soc Echocardiogr. 2015:28(1):1-39 e14.

24. Nagueh SF, Smiseth OA, Appleton CP, Byrd BF 3rd, Dokainish H, Edvardsen $T$, et al. Recommendations for the evaluation of left ventricular diastolic function by echocardiography: an update from the American Society of Echocardiography and the European Association of Cardiovascular Imaging. Eur Heart J Cardiovasc Imaging. 2016;17(12):1321-60.

25. Yingchoncharoen T, Agarwal S, Popovic ZB, Marwick TH. Normal ranges of left ventricular strain: a meta-analysis. J Am Soc Echocardiogr. 2013;26(2):185-91.

26. Voigt JU, Pedrizzetti G, Lysyansky P, Marwick TH, Houle H, Baumann R, et al. Definitions for a common standard for 2D speckle tracking echocardiography: consensus document of the EACVI/ASE/Industry Task Force to standardize deformation imaging. Eur Heart J Cardiovasc Imaging. 2015:16(1):1-11.

27. Myasoedova E, Davis JM 3rd, Crowson CS, Roger VL, Karon BL, Borgeson $\mathrm{DD}$, et al. Brief report: rheumatoid arthritis is associated with left ventricular concentric remodeling: results of a population-based cross-sectional study. Arthritis Rheum. 2013;65(7):1713-8.

28. Corrao S, Argano C, Pistone G, Messina S, Calvo L, Perticone F. Rheumatoid arthritis affects left ventricular mass: systematic review and meta-analysis. Eur J Intern Med. 2015;26(4):259-67.

29. Klocke R, Cockcroft JR, Taylor GJ, Hall IR, Blake DR. Arterial stiffness and central blood pressure, as determined by pulse wave analysis, in rheumatoid arthritis. Ann Rheum Dis. 2003;62(5):414-8.

30. Crilly MA, Kumar V, Clark HJ, Scott NW, Macdonald AG, Williams DJ. Arterial stiffness and cumulative inflammatory burden in rheumatoid arthritis: a dose-response relationship independent of established cardiovascular risk factors. Rheumatology (Oxford). 2009:48(12):1606-12.

31. Rudominer RL, Roman MJ, Devereux RB, Paget SA, Schwartz JE, Lockshin $M D$, et al. Independent association of rheumatoid arthritis with increased left ventricular mass but not with reduced ejection fraction. Arthritis Rheum. 2009;60(1):22-9.

32. Miyoshi T, Doi M, Hirohata S, Sakane K, Kamikawa S, Kitawaki T, et al. Cardioankle vascular index is independently associated with the severity of coronary atherosclerosis and left ventricular function in patients with ischemic heart disease. J Atheroscler Thromb. 2010;17(3):249-58.

33. Sato $Y$, Nagayama $D$, Saiki $A$, Watanabe $R$, Watanabe $Y$, Imamura $H$, et al. Cardio-ankle vascular index is independently associated with future cardiovascular events in outpatients with metabolic disorders. J Atheroscler Thromb. 2016;23(5):596-605.

34. Urbina EM, Dolan LM, McCoy CE, Khoury PR, Daniels SR, Kimball TR. Relationship between elevated arterial stiffness and increased left ventricular mass in adolescents and young adults. J Pediatr. 2011;158(5):715-21.

35. Chung CM, Lin YS, Chu CM, Chang ST, Cheng HW, Yang TY, et al. Arterial stiffness is the independent factor of left ventricular hypertrophy determined by electrocardiogram. Am J Med Sci. 2012;344(3):190-3.

36. Totaro S, Khoury PR, Kimball TR, Dolan LM, Urbina EM. Arterial stiffness is increased in young normotensive subjects with high central blood pressure J Am Soc Hypertens. 2015;9(4):285-92.

37. Thavendiranathan P, Poulin F, Lim KD, Plana JC, Woo A, Marwick TH. Use of myocardial strain imaging by echocardiography for the early detection of cardiotoxicity in patients during and after cancer chemotherapy: a systematic review. J Am Coll Cardiol. 2014;63(25 Pt A):2751-68.

38. Afonso LC, Bernal J, Bax JJ, Abraham TP. Echocardiography in hypertrophic cardiomyopathy: the role of conventional and emerging technologies. JACC Cardiovasc Imaging. 2008;1(6):787-800.

39. Midtbo H, Semb AG, Matre K, Kvien TK, Gerdts E. Disease activity is associated with reduced left ventricular systolic myocardial function in patients with rheumatoid arthritis. Ann Rheum Dis. 2017:76(2):371-6.

40. Castrejon I, Ortiz AM, Garcia-Vicuna R, Lopez-Bote JP, Humbria A, Carmona $L$, et al. Are the $C$-reactive protein values and erythrocyte sedimentation rate equivalent when estimating the 28 -joint disease activity score in rheumatoid arthritis? Clin Exp Rheumatol. 2008:26(5):769-75.

41. Fleischmann RM, van der Heijde D, Gardiner PV, Szumski A, Marshall L, Bananis E. DAS28-CRP and DAS28-ESR cut-offs for high disease activity in rheumatoid arthritis are not interchangeable. RMD Open. 2017;3(1):e000382.

42. Yiu KH, Tse HF, Mok MY, Lau CS. Ethnic differences in cardiovascular risk in rheumatic disease: focus on Asians. Nat Rev Rheumatol. 2011;7(10):609-18.

43. Buakhamsri A, Popovic ZB, Lin J, Lim P, Greenberg NL, Borowski AG, et al. Impact of left ventricular volume/mass ratio on diastolic function. Eur Heart J. 2009;30(10):1213-21

44. Manzi S, Meilahn EN, Rairie JE, Conte CG, Medsger TA Jr, Jansen-McWilliams $L$, et al. Age-specific incidence rates of myocardial infarction and angina in women with systemic lupus erythematosus: comparison with the Framingham study. Am J Epidemiol. 1997;145(5):408-15.

45. Mok CC, Kwok CL, Ho LY, Chan PT, Yip SF. Life expectancy, standardized mortality ratios, and causes of death in six rheumatic diseases in Hong Kong, China. Arthritis Rheum. 2011;63(5):1182-9.

46. Mor-Avi V, Lang RM, Badano LP, Belohlavek M, Cardim NM, Derumeaux G, et al. Current and evolving echocardiographic techniques for the quantitative evaluation of cardiac mechanics: ASE/EAE consensus statement on methodology and indications endorsed by the Japanese Society of Echocardiography. J Am Soc Echocardiogr. 2011;24(3):277-313.

47. Ikonomidis I, Tzortzis S, Lekakis J, Paraskevaidis I, Andreadou I, Nikolaou M, et al. Lowering interleukin-1 activity with anakinra improves myocardial deformation in rheumatoid arthritis. Heart. 2009;95(18):1502-7.

48. Spethmann S, Rieper K, Riemekasten G, Borges AC, Schattke S, Burmester $\mathrm{GR}$, et al. Echocardiographic follow-up of patients with systemic sclerosis by 2D speckle tracking echocardiography of the left ventricle. Cardiovasc Ultrasound. 2014:12:13.

\section{Publisher's Note}

Springer Nature remains neutral with regard to jurisdictional claims in published maps and institutional affiliations. 